\title{
Development of cockleshell (Anadara granosa) derived CaCO3 nanoparticle for doxorubicin delivery
}

\begin{abstract}
Despite the progress made in cancer treatment, difficulties are encountered with tumour targeting due to cancer structural complexity. The synthesis of homogenous calcium carbonate $(\mathrm{CaCO} 3)$ nanoparticles could be a carrier for doxorubicin in the management of bone cancer due to its osteoconductive and physicochemical properties with simple synthesis method to produce large scale. Among the nanocarriers, $\mathrm{CaCO} 3$ nanoparticles have exhibited promising potential as targeting drug nanocarrier. The aim of this study is to synthesised and characterised doxorubicin-conjugated $\mathrm{CaCO} 3$ nanoparticle (CS-CaCO3NP-DOX), using a simple precipitation and mechanical approach to synthesise homogeneous CaCO3NP from cockleshell. The oven-dried nanoparticles were further characterised for its physicochemical properties before and after conjugating with doxorubicin. A homogenous aragonite, spherical, porous nanocarrier was obtained with a mean diameter of $24.9 \mathrm{~nm}$ and zeta potential of -21 $\mathrm{mV}$. The energy dispersion X-ray analysis revealed high proportion of calcium as a major element in the nanoparticle. The spectrum peak suggests little alteration upon incorporation of doxorubicin. Higher loading content and encapsulation efficiency were recorded with CS$\mathrm{CaCO} 3 \mathrm{NP}$. These properties underscore the potential of $\mathrm{CS}-\mathrm{CaCO} 3 \mathrm{NP}$ in the delivery of doxorubicin, thus giving it a high potential for application in the delivery of the anticancer in the management of cancers.
\end{abstract}

Keyword: Cockleshell; Doxorubicin; Drug Delivery; Nanocarrier 